\title{
Simple and Effective Primary Assessment of Emergency Patients in a COVID-19 Outbreak Area: A Retrospective, Observational Study
}

This article was published in the following Dove Press journal: Risk Management and Healthcare Policy

\author{
Gaohong $\mathrm{Di}^{\prime}$ \\ Chengkun Xia' \\ Shanglong Yao' \\ Xiangdong Chen' \\ Zhilin Wu (D)' \\ Ling $\mathrm{Hu}^{2}$ \\ Jie Wang'
}

'Department of Anesthesiology, Union Hospital, Tongji Medical College,

Huazhong University of Science and Technology, Wuhan, Hubei, People's Republic of China; ${ }^{2}$ Department of Anesthesiology, Tianyou Hospital, Wuhan University of Science and Technology, Wuhan, Hubei, People's Republic of China
Correspondence: Jie Wang; Chengkun Xia Department of Anesthesiology of Wuhan Union Hospital, No. 1277 Jiefang Avenue, Hankou, Wuhan, Hubei, People's Republic of China

Tel/Fax +86 27-85726834

Email gatogiratorio@gmail.com;

xiayulong_200I@I63.com
Background: The rapid spread of COVID-19 has expanded into a pandemic, for which the main containment strategies to reduce transmission are social distancing and isolation of ill persons. Thousands of medical staff have been infected worldwide. Coronavirus testing kits have been in short supply, and early diagnostic reagents did not have high sensitivity. The aim of this study was to describe the characteristics of patients requiring emergency surgery in a COVID-19 outbreak area.

Methods: We assessed medical data regarding all patients who underwent emergency surgery at the main campus of Wuhan Union Hospital from January 23, 2020, to February 15, 2020. We classified patients based on suspicion of COVID-19 infection (suspected vs not suspected) before they were admitted to the operating room. We used descriptive statistics to analyze the data. Outcomes included the incidence of confirmed COVID-19 infection and length of stay, which were followed until March 25, 2020.

Results: Among the 88 emergency patients included in this study, the mean age was 37 years. Twenty-five patients presented with abnormalities observed on chest CT scans and 16 presented with fever. The median wait time for surgery was one day. The median preparation time and median time until short orientation memory concentration test (SOMCT) recovery from anesthesia were $44.0 \mathrm{~min}$ and $23.0 \mathrm{~min}$, respectively. The median postoperative length of stay was five days. Compared with patients not suspected of COVID-19 infection, six patients were confirmed to be infected with COVID-19 in the suspected group. No health care workers were infected during this study period.

Conclusion: Simple identification using temperature screening of patients, respiratory symptoms, and chest CT scans before being admitted for emergency surgery was rapid and effective. Shortened contact times might reduce the risk of infection. Additional investigations with larger samples and improved designs are needed to confirm these observations.

Keywords: COVID-19, emergency, cross-infection

\section{Introduction}

In December 2019, a series of pneumonia cases with clinical presentations that resembled severe acute respiratory syndrome coronavirus (SARS-CoV) and Middle East respiratory syndrome coronavirus (MERS-CoV), emerged in Wuhan, Hubei, China. ${ }^{1,2}$ A Chinese scientific team subsequently discovered a virus in the subgenus Sarbecovirus of the genus Betacoronavirus in those patients. ${ }^{3}$ The pandemic is now named coronavirus disease-2019 (COVID-19). The rapid spread of COVID-19 expanded into a pandemic in March 2020. ${ }^{4}$ Previous studies have described the epidemiological and clinical characteristics of patients with COVID-19 
pneumonia. ${ }^{5-8}$ Currently, the main containment strategies to reduce transmission are social distancing and isolating ill persons. ${ }^{9}$ However, infection with COVID-19 has been reported in health care workers in hospitals. ${ }^{8,10}$ Thus, all public health care systems are facing evolving critical challenges globally.

Many guidelines have been drafted to prevent the nosocomial spread of COVID-19. ${ }^{11,12}$ However, in the early period of the infection, patients often showed only mild symptoms or were asymptomatic, which increases the difficulty in identifying patients with COVID-19 infections. This was especially true when identifying patients who needed emergency surgery in the hospital located in the initial outbreak area. Moreover, the positive diagnosis rates using qRT-PCR did not exhibit high sensitivity in clinical settings due to the use of different inactivation methods. $^{13}$ The aim of this study was to describe the clinical, laboratory, and radiological characteristics of patients who underwent emergency surgery and compare the clinical features between patients suspected of being infected with COVID-19 to patients who were not suspected of infection. Moreover, with consideration of possible shortages in medical supplies, we designed protective measures based on suspicion of COVID-19 infection to prevent cross-infection in the operating room.

\section{Materials and Methods Study Design}

This was a single-center, retrospective, observational study that was conducted on the main campus of Wuhan Union Hospital (Wuhan, China), one of the major tertiary teaching hospitals in the endemic area of COVID-19. We analyzed patients who underwent emergency surgery at the main hospital campus from January 23, 2020, to February 15, 2020. Emergency surgery was defined as surgery required to deal with an acute threat to life, organ, limb, or tissue, which needed to be addressed immediately. All of Wuhan's public transportation was suspended on January 23, 2020. Subsequently, the main campus of Wuhan Union Hospital suspended all elective surgeries. Before February 15, 2020, we did not perform throat swab tests on all emergency patients to screen for COVID-19 infection at the time of admission, due to a lack of diagnostic reagents. The qRT-PCR results were obtained after 12 hours. Because the patient's condition was urgent, all the surgeries could not be postponed until the qRT-PCR results were received. The outcomes of this study included confirmed COVID-19 cases and the length of stay in the hospital, which were monitored until March 25, 2020. During the outbreak, the emergency department of the main hospital campus was responsible for receiving all patients in Wuhan who required emergency surgery. All study methods were conducted following the relevant regulations and guidelines of the institutional ethics committee of Tongji Medical College, Huazhong University of Science and Technology. Written informed consent was waived due to the rapidly emerging infectious disease.

\section{Data Collection}

The electronic medical records of patients were analyzed by members of the anesthesiology department at Wuhan Union Hospital. We reviewed clinical, laboratory, and radiological characteristics and nursing records. The information that was recorded included demographic data (age and sex), medical history (chronic medical histories), symptoms, clinical signs (temperature), laboratory findings (white blood cell count, lymphocyte count, lymphocyte percentage, C-reactive protein (CRP), and highsensitivity C-reactive protein (hr-CRP)), chest computed tomographic (CT) scans, and anesthesia records (ASA scores, type of anesthesia, type of surgery, and perioperative time points).

Skilled anesthesiologists determined scores based on criteria from the American Society of Anesthesiologists (ASA) scoring system. Abnormalities observed on chest CT scans included ground-glass opacity, patchy local shadowing, or other inflammatory signs present in a patient's lungs. The wait time for surgery was calculated from admission to the day of surgery. The surgical preparation time was defined as the duration between the time the patient entered the operating room and the time of the initial incision. The time until short orientation memory concentration test (SOMCT) recovery was defined as the duration between the closure of the incision and the time the patient exited the operating room.

We classified patients based on suspicion of COVID19 infection (suspected vs not suspected). Suspicion of COVID-19 infection was defined as patients having a temperature higher than $37.2^{\circ} \mathrm{C}$ or an abnormal chest $\mathrm{CT}$ before entering the operating room. We defined a patient as not suspected of COVID-19 infection when their temperature and CT scans were normal before entering the operating room (if CT scans were available). 


\section{Statistical Analysis}

Continuous variables including age, preparation time, wait times for surgery, time for SOMCT recovery, postoperative CRP, postoperative hs-CRP, and length of stay were expressed as medians (and interquartile ranges) or means (and standard deviation). The remaining data were categorical variables, which were summarized as counts and percentages. Differences between suspected and not suspected COVID-19 infections were calculated using the Mann-Whitney-Wilcoxon test or a two-sample $t$-test for continuous variables and the chi-square test or Fisher's Exact test for categorical variables. A two-sided $\alpha$ of less than 0.05 was considered to be statistically significant. No imputation was made for missing data. The data analysis was performed using $\mathrm{R}$ software, version 3.6.3 (R Foundation for Statistical Computing).

\section{Results}

Between January 23, 2020, and February 15, 2020, the surgery department admitted 88 patients with indications for emergency surgery. Fourteen patients suffered from external trauma, 12 presented with acute abdomen, four with aortic dissection, two with critical coronary heart disease (CHD), four with esophageal foreign bodies, 27 with fetal distress, 14 with placental abruption, five with hemorrhage in placenta previa, four with globe rupture, one with pneumothorax, and one with testicular torsion. Five patients exhibited a mild cough, none of the patients presented obvious acute respiratory infection symptoms, such as a continuous cough, nasal congestion, sore throat, or headache. Thus, all 88 patients were included in this study.

\section{Preoperative Characteristics}

Of the 88 patients, 11 (12.5\%) were older than 60 years, and seven $(7.9 \%)$ were less than 16 years. The mean patient age was 37 years (SD, 17.8 years). Sixty-three (71.6\%) patients were female, and $16(18.2 \%)$ patients had a body temperature higher than $37.2^{\circ} \mathrm{C}$ before entering the operating room. Thirty-five patients had chest CT scans available, and of those 35 patients, four presented images with ground-glass opacity, eight presented with patchy local shadowing, 13 presented with patchy bilateral shadowing, and 10 presented with normal CT scans. Five patients exhibited a mild cough, and their CT scans were abnormal. Leucocytes were below the normal range in four $(4.7 \%)$ patients and above the normal range in 27
(31.8\%) patients. Lymphocytopenia was present in 22 (25.9\%) patients. Twenty-five patients exhibited elevated levels of CRP or hs-CRP. (Table 1)

\section{Surgery Characteristics}

Of the 88 cases, nine emergency surgeries were repeat surgeries for disease progression in patients who had been hospitalized before January 23, 2020. Thirty-two (36.4\%) patients received surgery on the same day as they were admitted, and $24(27.3 \%)$ patients went to surgery on the second day after admission. The medical conditions for the majority of patients $(64.8 \%)$ were classified as ASA I and II. The median surgical preparation time was less than one hour (44 minutes). More than half of the patients (55.7\%) underwent non-general anesthesia and did not undergo tracheal intubation. Of the 31 patients who were extubated and recovered from general anesthesia in the operating room, the median time for SOMCT recovery was less than half an hour (23 minutes). Obstetric surgery accounted for $52.3 \%$ of the surgical cases. Eight patients were transferred to the intensive care unit due to poor postoperative conditions. (Table 2)

\section{Postoperative Characteristics}

Within seven days postoperatively, the highest temperature observed in 18 patients $(20.5 \%)$ was higher than $37.2^{\circ} \mathrm{C}$. Over half of the patients (59.3\%) in this study exhibited leukocytosis, and $66.7 \%$ of the patients exhibited lymphocyte counts in the normal range. In patients with elevated CRP and hs-CRP levels, which are biomarkers associated with infection, the mean CRP and hs-CRP levels were 82.2 and 78, respectively. Of the patients suspected of viral infection, six patients were confirmed to be positive for COVID-19 during the postoperative period. No patients who were initially classified as not suspected of viral infection tested positive for COVID-19. No anesthesiologists or nurses who participated in the emergency surgeries during this study showed respiratory symptoms in the 14 days following contact with any of the patients. Seven patients were still hospitalized on the final day of the follow-up period used in this study. The median duration of the postoperative length of stay was five days (Table 3).

\section{Discussion}

COVID-19 can be transmitted through aerosols and small droplets from normal breathing, coughing, sneezing, or fluids from human secretions. ${ }^{7}$ The virus is highly 
Table I Preoperative Characteristics of Study Patients with Emergency Surgery, According to Identification of Suspicious for COVID-19

\begin{tabular}{|c|c|c|c|c|}
\hline Characteristic & All Patients $(\mathrm{N}=\mathbf{8 8})$ & Non-Suspected $(n=52)$ & Suspected $(n=36)$ & $P$ value* \\
\hline Age, mean (SD), y & $37.1(17.8)$ & $32.7(13.0)$ & $43.6(21.7)$ & $<0.001$ \\
\hline \multicolumn{5}{|l|}{ Sex } \\
\hline Female & $63 / 88(71.6 \%)$ & $45 / 52(86.5 \%)$ & $18 / 36(50.0 \%)$ & $<0.001$ \\
\hline Male & $25 / 88(28.4 \%)$ & $7 / 52(13.5 \%)$ & $18 / 36(50.0 \%)$ & \\
\hline \multicolumn{5}{|l|}{ Comorbidities } \\
\hline Hypertension & $3 / 88$ (3.4\%) & $1 / 52$ (1.9\%) & $2 / 36(5.6 \%)$ & 0.565 \\
\hline Diabetes & $3 / 88(3.4 \%)$ & $0 / 52$ & $3 / 36(8.3 \%)$ & 0.06507 \\
\hline Temperature $>37.2^{\circ} \mathrm{C}$ & $16 / 88(18.2 \%)$ & $0 / 52$ & $16 / 36(44.4 \%)$ & $<0.001$ \\
\hline Abnormal on chest $\mathrm{CT}$ & $25 / 35(71.4 \%)$ & $0 / 8$ & $25 / 27(92.6 \%)$ & $<0.001$ \\
\hline \multicolumn{5}{|l|}{ Laboratory findings** } \\
\hline \multicolumn{5}{|l|}{ White blood cell count, $\times 10^{9}$ per $\mathrm{L}$} \\
\hline $4-10$ & $54 / 85(63.5 \%)$ & $37 / 49(75.5 \%)$ & $17 / 36(47.2 \%)$ & 0.01901 \\
\hline$<4$ & $4 / 85(4.7 \%)$ & $1 / 49(2.0 \%)$ & $3 / 36(8.3 \%)$ & \\
\hline$>10$ & $27 / 85(31.8 \%)$ & I I/49 (22.4\%) & $16 / 36(44.4 \%)$ & \\
\hline \multicolumn{5}{|l|}{ Lymphocyte count, $\times 10^{9}$ per $\mathrm{L}$} \\
\hline $1.1-3.2$ & $59 / 85(69.4 \%)$ & $40 / 49(81.6 \%)$ & $19 / 36(52.8 \%)$ & 0.01208 \\
\hline$<1.1$ & $22 / 85(25.9 \%)$ & $8 / 49(16.3 \%)$ & $14 / 36(38.9 \%)$ & \\
\hline$>3.2$ & $4 / 85(4.7 \%)$ & $1 / 49(2.0 \%)$ & $3 / 36(8.3 \%)$ & \\
\hline \multicolumn{5}{|l|}{ Lymphocytes percentage } \\
\hline $20 \%-40 \%$ & $26 / 85(30.6 \%)$ & $21 / 49(42.9 \%)$ & $5 / 36(13.9 \%)$ & 0.00865 \\
\hline$<20 \%$ & $59 / 85(69.4 \%)$ & $28 / 49(57.1 \%)$ & $31 / 36(86.1 \%)$ & \\
\hline$C R P>3.14 \mathrm{mg} / \mathrm{L}$ or hs-CRP> $10 \mathrm{mg} / \mathrm{dL}$ & $25 / 68(36.8 \%)$ & $9 / 43(20.9 \%)$ & $16 / 25(64.0 \%)$ & $<0.001$ \\
\hline
\end{tabular}

Notes: $* P$ values indicate differences between Non-suspected and Suspected patients. $P<0.05$ was considered statistically significant.** Children" value are classified according to pediatric normal ranges.

contagious and has led to a global pandemic. The ability to screen and identify patients infected with COVID-19 that were admitted to the emergency department at the main campus of Wuhan Union Hospital was complicated by the variability in the clinical presentation of infected individuals. However, fever, cough, and radiologic abnormalities have been identified as the dominant symptoms in patients infected with COVID-19. ${ }^{6}$ During this study, no chest CT scans or throat swabs were taken as routine COVID-19 screening measures for emergency surgery patients due to the shortage of medical resources. Therefore, we designed a simple management process to prevent nosocomial infections. We rapidly and effectively classified patients through the use of chest $\mathrm{CT}$ scans and monitoring their body temperature. Because the hospital was located in the original outbreak area, it was likely that asymptomatic patients and patients in the viral incubation period (without fever or radiologic abnormalities) were admitted to the hospital. ${ }^{2}$ Therefore, all medical staff wore N95 respirators as a necessary protective measure. Additional high-level protection measures were implemented when treating patients suspected to be infected, including wearing a face shield, medical goggles, and disposable protective clothing. Although it has been reported there is no difference between wearing N95 respirators and surgical masks, ${ }^{14}$ the potential for respiratory tract infection by COVID-19 was considered to be greater than other viruses. Therefore, N95 respirators were fit-tested to prevent virus transmission effectively.

In our study, more female patients were involved because of the number of emergency cesarean sections $(52.3 \%)$ that were needed. Based on the preoperative laboratory findings, patients suspected of COVID-19 infection exhibited abnormal leukocyte counts, lymphocyte counts, and CRP (hs-CRP) compared to patients who were not suspected to be infected. Many previous reports have revealed that lymphocytopenia is common in COVID-19 cases. ${ }^{2,5}$ However, most emergency patients present with fever and leukocytosis, which made it more challenging to screen and identify atypical patients, 
Table 2 Surgery Characteristics of Study Patients with Emergency Surgery

\begin{tabular}{|c|c|c|c|c|}
\hline Characteristic & All Patients $(\mathbf{N}=\mathbf{8 8})$ & Non-Suspected $(n=52)$ & Suspected $(n=36)$ & $P$ value \\
\hline Reoperation for inpatients & $9 / 88(10.2 \%)$ & $2 / 52(3.8 \%)$ & $7 / 36(19.4 \%)$ & 0.02873 \\
\hline Median waiting times for surgery (IQR), d & $1.00(3.00)$ & $1.00(2.00)$ & $1.50(7.25)$ & 0.05294 \\
\hline \multicolumn{5}{|l|}{ ASA scores } \\
\hline I & $3 / 88(3.4 \%)$ & $1 / 52(1.9 \%)$ & $2 / 36(5.6 \%)$ & 0.00374 \\
\hline II & $54 / 88(61.4 \%)$ & $40 / 52(76.9 \%)$ & $14 / 36(38.9 \%)$ & \\
\hline III & $20 / 88(22.7 \%)$ & $8 / 52(15.4 \%)$ & $12 / 36(33.3 \%)$ & \\
\hline IV & II/88 (12.5\%) & $3 / 52(5.8 \%)$ & $8 / 36(22.2 \%)$ & \\
\hline Median prepare time (IQR), min & $44.0(20.0)$ & $41.5(18.0)$ & $46.0(37.0)$ & 0.1039 \\
\hline \multicolumn{5}{|l|}{ Anesthesia technique } \\
\hline General anesthesia & $39 / 88(44.3 \%)$ & $10 / 52(19.2 \%)$ & $29 / 36(80.6 \%)$ & $<0.001$ \\
\hline Non-general anesthesia & $49 / 88(55.7 \%)$ & $42 / 52(80.8 \%)$ & $7 / 36(19.4 \%)$ & \\
\hline Emergence in OR & $31 / 88(35.2 \%)$ & $9 / 52(17.3 \%)$ & $22 / 36(61.1 \%)$ & $<0.001$ \\
\hline Median time until SOMCT recovery (IQR), min & $23.0(16.5)$ & $23.0(2.00)$ & $22.0(24.0)$ & 0.5855 \\
\hline Cases transferred to ICU & $8 / 88(9.1 \%)$ & $1 / 52(1.9 \%)$ & $7 / 36(19.4 \%)$ & 0.00722 \\
\hline \multicolumn{5}{|l|}{ Surgical specialty } \\
\hline Gastrointestinal & $12 / 88(13.6 \%)$ & $3 / 52(5.8 \%)$ & $9 / 36(25.0 \%)$ & $<0.001$ \\
\hline Cardiac & $6 / 88(6.8 \%)$ & $0 / 52$ & $6 / 36(16.7 \%)$ & \\
\hline Obstetrics & $46 / 88(52.3 \%)$ & $39 / 52(75.0 \%)$ & $7 / 36(19.4 \%)$ & \\
\hline Thoracic & $2 / 88(2.3 \%)$ & $0 / 52$ & $2 / 36(5.6 \%)$ & \\
\hline Otorhinolaryngology & $3 / 88(3.4 \%)$ & $1 / 52(1.9 \%)$ & $2 / 36(5.6 \%)$ & \\
\hline Ophthalmology & $4 / 88(4.5 \%)$ & $1 / 52(1.9 \%)$ & $3 / 36(8.3 \%)$ & \\
\hline Traumatic & |4/88 (15.9\%) & $8 / 52(15.4 \%)$ & $6 / 36(16.7 \%)$ & \\
\hline Pediatric & $\mathrm{I} / 88(\mathrm{I} .1 \%)$ & $0 / 52$ & $1 / 36(2.8 \%)$ & \\
\hline
\end{tabular}

Table 3 Postoperative Characteristics of Study Patients with Emergency Surgery

\begin{tabular}{|c|c|c|c|c|}
\hline Characteristic & All Patients $(\mathrm{N}=\mathbf{8 8})$ & Non-Suspected $(n=52)$ & Suspected $(n=36)$ & $P$ value \\
\hline \multicolumn{5}{|l|}{ Within 7 days postoperatively } \\
\hline Highest temperature $>37.2^{\circ} \mathrm{C}$ & I8/88 (20.5\%) & $5 / 52(9.6 \%)$ & 13/36 (36.1\%) & 0.00576 \\
\hline \multicolumn{5}{|l|}{ Mean white blood cell count, $\times 10^{9} / \mathrm{L}$} \\
\hline $4-10$ & $33 / 81$ (40.7\%) & I6/47 (34.0\%) & $17 / 34(50.0 \%)$ & 0.225 \\
\hline$>10$ & $48 / 81$ (59.3\%) & $31 / 47(66.0 \%)$ & $17 / 34(50.0 \%)$ & \\
\hline \multicolumn{5}{|l|}{ Mean lymphocyte count, $\times 10^{9} / \mathrm{L}$} \\
\hline I.I-3.2 & $54 / 81(66.7 \%)$ & $39 / 47$ (83.0\%) & I5/34 (44.I\%) & $<0.001$ \\
\hline$<1.1$ & $26 / 81$ (32.1\%) & $7 / 47$ (14.9\%) & 19/34 (55.9\%) & \\
\hline$>3.2$ & $\mathrm{I} / 8 \mathrm{I}(\mathrm{I} .2 \%)$ & I/47 (2.1\%) & $0 / 34$ & \\
\hline \multicolumn{5}{|l|}{ Mean lymphocytes percentage } \\
\hline $20 \%-40 \%$ & $|4 / 8|(\mid 7.3 \%)$ & $8 / 47(17.0 \%)$ & $6 / 34(17.6 \%)$ & 0.693 \\
\hline$<20 \%$ & $66 / 81$ (8I.5\%) & $38 / 47$ (80.9\%) & $28 / 34(82.4 \%)$ & \\
\hline$>40 \%$ & $\mathrm{I} / 8 \mathrm{I}(1.2 \%)$ & $1 / 47(2.1 \%)$ & $0 / 34$ & \\
\hline CRP or hs-CRP available & 77/88 (87.5\%) & $47 / 52(90.4 \%)$ & $30 / 36(83.3 \%)$ & 0.3461 \\
\hline Mean CRP (SD), mg/L & $82.2(59.5)$ & $57.3(45.7)$ & $92.2(62.7)$ & 0.233 \\
\hline Mean hs-CRP (SD), mg/dL & $78.0(49.2)$ & $78.0(49.5)$ & $78.0(50.0)$ & 0.9983 \\
\hline Confirmed COVID-19 Cases & $6 / 88(6.8 \%)$ & $0 / 52$ & $6 / 36(16.7 \%)$ & 0.00359 \\
\hline Median postoperative length of stay (IQR), $d$ & $5.00(4.00)$ & $5.00(2.00)$ & $7.00(5.50)$ & 0.03535 \\
\hline
\end{tabular}


asymptomatic patients, and patients who were still in the incubation period for COVID-19 infection.

All patients wore standard surgical masks before they were transported into the preoperative preparation room. Airway assessment was done by inquiry instead of inspection with the mask removed. At least two different tracheal intubation devices were provided to avoid difficulty during tracheal intubation. Fortunately, no patient needed secondary tracheal intubation in this study. The method of anesthesia that was selected depended on the specific surgical requirements and the condition of the patient. Non-general anesthesia patients wore a surgical mask throughout the surgery. We then placed an anesthetic face mask, which was connected to the anesthesia machine, to deliver oxygen through the patient's surgical mask. For general anesthesia, video laryngoscope lenses were applied, which maximized the distance between the operator's and patient's faces to reduce the risk of viral transmission. Extubation was performed when the patient was fully awake. After extubation, we placed an anesthetic face mask on the patient's face to deliver oxygen and replaced the patient's surgical mask immediately when the recovery conditions met the standards needed to allow the patient to leave the operating room. Emergence was performed in the operating room instead of the postanesthesia care unit. In our study, the median preparation time and median time until SOMCT recovery were $44 \mathrm{~min}$ and $23 \mathrm{~min}$, respectively. Therefore, minimizing contact time with patients should reduce the risk of exposure. The anesthetic machine and operating room were disinfected immediately after each surgery. Effective disinfection of the anesthesia machine and operating room were essential measures to ensure that the next surgical patient was not infected by virus possibly left by the previous patient. All patients were placed in separate rooms postoperatively to prevent any cross-infection in the ward after surgery.

When patients suspected to be infected with COVID19 were compared to non-suspected patients, more suspected patients presented with fever and lymphocytopenia. However, the CRP or hs-CRP values were not significantly different between the two patient groups. Fever is commonly observed during the immediate and early postoperative periods following surgery, and nonsteroidal antiinflammatory drugs are routine postoperative medications used to adjust the patient's body temperature. ${ }^{15}$ Therefore, postoperative medications and surgical wound inflammation might influence some laboratory results, even if the patient had a viral infection.
The COVID-19 cases in this study were confirmed in the postoperative period using chest $\mathrm{CT}$ scans and throat swabs that detected viral nucleic acid using qRT-PCR assays. Six patients were confirmed to be infected with COVID-19, and all six patients were in the suspected group, four of them presented images with ground-glass opacity in preoperative period. This result indicated that the preoperative classification system used with emergency patients based on body temperature measurements and chest $\mathrm{CT}$ scans was effective for patients who presented mild symptoms or were the incubation period. It has been reported that the incubation period of COVID-19 is estimated to be five days. ${ }^{16}$ However, no patients presented any acute respiratory symptoms in the nonsuspected group. Not all patients underwent throat swab screening, so we could not confirm the existence of any asymptomatic patients. Fortunately, no fever and or respiratory symptoms appeared in any of the anesthesiologists and nurses who collaborated in the emergency surgeries conducted during this study. Seven patients were still hospitalized due to surgical complications at the end of this study. All confirmed cases of COVID-19 viral infection were transferred to a COVID-19 isolation ward.

Although this was a single-center study with a relatively small number of cases, the procedures implemented to identify patients suspected of COVID-19 infection and the application of appropriate protective measures were effective. However, we note that every hospital has developed a management strategy to deal with the viral outbreak. We suggest that throat swabs or other COVID19 tests be performed as soon as possible after patients are admitted to the emergency department, to optimize the identification of COVID-19 infection. Some laboratory results might be influenced or masked by the patient's perioperative condition and postoperative medications. In Wuhan, the epicenter of the viral outbreak, we lacked many of the necessary protective resources at the beginning of the disease outbreak. Through our established screening methods, we could save many resources needed to process situations that also needed protection. The rational use of protective resources after patients are classified based on suspicion of viral infection might alleviate the lack of protection caused by shortages in protective resources.

This study had several limitations. First, some cases had incomplete laboratory testing due to the short length of stay of the patient. For example, the cases involving 
a removal procedure carried out under painless gastrointestinal endoscopy did not require lengthy post-surgical stays. Second, preoperative chest CT scans were not obtained for all surgical patients due to the severe nature of some patients' conditions. Third, the throat swabs used to detect viral nucleic acid were not obtained from all patients included in this study. There was a shortage of diagnostic reagents during the study period, so we could not assess the asymptomatic cases. Fourth, this was a single-center study with a small sample size. A larger sample size might result in more pronounced results between the two groups.

\section{Conclusion}

In the circumstances of a patient's emergency condition and lack of sufficient diagnostic reagents for COVID-19, identification of patients with possible viral infection using temperature screening, the presence or absence of respiratory symptoms, and chest CT scans was rapid and effective. Reducing the patients' respiratory exposure time and contact time with other individuals might reduce the risk of cross-infection between the medical staff and patients.

\section{Abbreviations}

COVID-19, coronavirus disease-2019; qRT-PCR, quantitative real-time polymerase chain reaction; $\mathrm{SD}$, standard deviation; ASA, American Society of Anesthesiologists; CT, computed tomography; SOMCT, short orientation memory concentration test; CHDs, congenital heart disease; CRP, C-reactive protein; hs-CRP, high-sensitivity C-reactive protein; IQR, interquartile range; GA, general anesthesia; ICU, intensive care unit.

\section{Ethics Approval and Consent to Participate}

Tongji Medical College Ethics Committee approved our observational study. We were compliant with the Declaration of Helsinki, with respect to ethical treatment of patients in this clinical retrospective study. This study used only anonymized data and the rapid emergence of this infectious disease, written informed consent was waived.

\section{Acknowledgments}

The authors thank all health care workers to fight the epidemic outbreak for their courageous work.

\section{Author Contributions}

All authors made a significant contribution to the work reported, whether that is in the conception, study design, execution, acquisition of data, analysis and interpretation, or in all these areas; took part in drafting, revising or critically reviewing the article; gave final approval of the version to be published; have agreed on the journal to which the article has been submitted; and agree to be accountable for all aspects of the work.

\section{Funding}

Funded by the Natural Science Foundation of Hubei Province ZRMS2019000029. The source of funding of the authors playing no role in the design of the study and collection, analysis, and interpretation of data and in writing the manuscript.

\section{Disclosure}

The authors report no conflicts of interest in this work.

\section{References}

1. Pneumonia of unknown cause China: disease outbreak news. Geneva: World Health Organization. Available from:. https://www.who.int/ csr/don/05-january-2020-pneumonia-of-unkown-cause-china/en/. Accessed January 5, 2020.

2. Huang $\mathrm{C}$, Wang $\mathrm{Y}, \mathrm{Li} \mathrm{X}$, et al. Clinical features of patients infected with 2019 novel coronavirus in Wuhan, China. Lancet. 2020;395:497-506. doi:10.1016/S0140-6736(20)30183-5

3. Lu R, Zhao X, Li J, et al. Genomic characterization and epidemiology of 2019 novel coronavirus: implications of virus origins and receptor binding. Lancet. 2020;395:565-574. doi:10.1016/S0140-6736(20)30251-8

4. Coronavirus disease (COVID-19) situation dashboard Available from: https://experience.arcgis.com/experience/ 685d0ace521648f8a5beeee1b9125cd.

5. Chen N, Zhou M, Dong X, et al. Epidemiological and clinical characteristics of 99 cases of 2019 novel coronavirus pneumonia in Wuhan, China: A descriptive study. Lancet. 2020;395:507-513. doi:10.1016/S0140-6736(20)30211-7

6. Guan WJ, Ni ZY, Hu Y, et al. Clinical characteristics of coronavirus disease 2019 in China. $N$ Engl J Med. 2020;382:1708-1720. doi:10.1056/NEJMoa2002032

7. Wang D, Hu B, Hu C, et al. Clinical characteristics of 138 hospitalized patients with 2019 novel coronavirus-infected Pneumonia in Wuhan, China. JAMA. 2020;323:1061. doi:10.1001/jama.2020.1585

8. Li Q, Guan X, Wu P, et al. Early transmission dynamics in wuhan, china, of novel coronavirus-infected Pneumonia. $N$ Engl J Med. 2020;382(13):1199-1207. doi:10.1056/NEJMoa2001316

9. Fauci AS, Lane HC, Redfield RR. COVID-19 navigating the uncharted. $N$ Engl J Med. 2020;382(13):1268-1269. doi:10.1056/ NEJMe2002387

10. The Lancet. COVID-19: protecting health-care workers. Lancet. 2020;395(10228):922. doi:10.1016/S0140-6736(20)30644-9.

11. Strategies for optimizing the supply of PPE. https:/www.cdc.gov/ coronavirus/2019-ncov/hcp/ppe-strategy/index.html.

12. Chen X, Liu Y, Gong Y, et al. Perioperative management of patients infected with the Novel Coronavirus: recommendation from the joint task force of the chinese society of anesthesiology and the Chinese association of anesthesiologists. Anesthesiology. 2020;132:13 07-1316. doi:10.1097/ALN.0000000000003301 
13. Pan Y, Long L, Zhang D, et al. Potential false-negative nucleic acid testing results for severe acute respiratory syndrome coronavirus 2 from thermal inactivation of samples with low viral loads. Clin Chem. 2020:pii: hvaa091. doi:10.1093/clinchem/hvaa091.

14. Radonovich LJ Jr, Simberkoff MS, Bessesen MT, et al. N95 respirators vs medical masks for preventing Influenza among health care personnel: a randomized clinical trial. JAMA. 2019;322(9):824-833. doi:10.1001/jama.2019.11645
15. Murphy PJ, Myers BL, Badia P. Nonsteroidal anti-inflammatory drugs alter body temperature and suppress melatonin in humans. Physiol Behav. 1996;59(1):133-139. doi:10.1016/0031-9384(95)02036-5

16. Lauer SA, Grantz KH, Bi Q, et al. The incubation period of Coronavirus disease 2019 (COVID-19) from publicly reported confirmed cases: estimation and application. Ann Intern Med. 2020;172:577-582. doi:10.7326/M20-0504

\section{Publish your work in this journal}

Risk Management and Healthcare Policy is an international, peerreviewed, open access journal focusing on all aspects of public health, policy, and preventative measures to promote good health and improve morbidity and mortality in the population. The journal welcomes submitted papers covering original research, basic science, clinical \& epidemiological studies, reviews and evaluations, guidelines, expert opinion and commentary, case reports and extended reports. The manuscript management system is completely online and includes a very quick and fair peer-review system, which is all easy to use. Visit http://www.dovepress.com/testimonials.php to read real quotes from published authors. 